\title{
Qualidade de Vida e Condições de Higiene de Próteses Dentárias de Idosos Institucionalizados
}

\section{Quality of Life and Hygiene Conditions of Dental Prostheses of Institutionalized Elderly People}

\author{
Tainá Nascimento Falcão ${ }^{1}$ \\ Mariana Mélani Alexandrino Costa ${ }^{1}$ \\ Loyse Martorano Fernandes ${ }^{2}$ \\ Leopoldina de Fátima Dantas de Almeida ${ }^{3}$ \\ Ana Maria Gondim Valença ${ }^{4}$
}

\section{RESUMO}

Objetivo: Avaliou-se a qualidade de vida e as condições de higiene de próteses dentárias em idosos residentes em instituição de longa permanência, na Cidade de João Pessoa-PB. Metodologia: Foi aplicado o Geriatric Oral Health Assessment Index (GOHAI) para avaliar a qualidade de vida, em alta e moderada-baixa, incluindo perguntas sobre higienização de prótese dentária $(n=38)$. Adicionalmente, as próteses de cada indivíduo foram recobertas por solução evidenciadora e fotografadas, para mensuração do biofilme. Os dados foram analisados inferencialmente a partir dos testes Qui-quadrado e Exato de Fisher $(\alpha=5 \%)$. A retenção do biofilme foi classificada, a partir das áreas coradas, em alta, média e baixa. Resultados: A média de idade da amostra foi de $81,8 \pm 7,7$ anos, sendo $86,8 \%(n=33)$ do sexo feminino. Apenas $34,2 \%$ afirmaram ter recebido instrução sobre higienização e $86,8 \%$ referiram não remover a prótese em nenhum período do dia. A qualidade de vida não esteve associada ao tempo de uso da prótese, ou com o conhecimento em relação aos métodos químicos e mecânicos de higienização $(p>0,05)$, mas se mostrou alta quando associada ao fato de não retirarem a prótese para dormir $(p<0,05)$. Dentre as 26 próteses superiores analisadas, em $53,8 \%(n=14)$ observou-se baixa retenção; quanto às 13 próteses inferiores avaliadas, $53,8 \%(n=7)$ apresentaram retenção moderada. Conclusão: A alta qualidade de vida esteve associada ao fato do idoso não retirar a prótese para dormir. As próteses inferiores apresentaram maior retenção de biofilme em relação às próteses superiores.

DESCRITORES: Prótese dentária. Higiene Bucal. Saúde Bucal.

\begin{abstract}
Objetive: Quality of life and hygiene conditions of dental prostheses were evaluated in elderly residents of a long-term institution in the city of João Pessoa-PB. Methods: The Geriatric Oral Health Assessment Index (GOHAI) was used to evaluate high and moderate-low quality of life, including dental hygiene questions $(n=38)$. In addition, the prostheses of each individual were covered by an evidentiary solution and photographed for biofilm measurement. Data were analyzed inferentially from Chisquare and Fisher's exact tests ( $\alpha=5 \%)$. The retention of the biofilm was classified, from the stained areas, in high, medium and low. Results: The mean age of the sample was 81.8 years $( \pm 7.7)$, of which $86.8 \%(n=33)$ were female. Only $34.2 \%$ reported having received instruction on hygiene and $86.8 \%$ reported not removing the prosthesis at any time of the day. The quality of life was not associated with the time of use of the prosthesis, or with the knowledge regarding the chemical and mechanical methods of hygiene $(p>0.05)$, but it was high when associated with the fact that they did not remove the prosthesis to sleep $(p<0.05)$. Among the 26 upper prostheses analyzed in $53.8 \%$ $(n=14)$, low retention was observed; regarding the 13 inferior prostheses evaluated, $53.8 \%(n=7)$ presented moderate retention. Conclusion: The high quality of life was associated with the fact that the elderly did not remove the prosthesis to sleep. The lower prostheses presented higher retention of biofilm in relation to the upper prostheses.
\end{abstract}

DESCRIPTORS: Dental Prosthesis. Oral Hygiene. Oral Health.

1- Acadêmica do Curso de Odontologia, Universidade Federal da Paraíba - UFPB, João Pessoa, Paraíba, Brasil.

2- Doutoranda do programa de Pós-Graduação em Clínica Odontológica (Prótese Dentária), Faculdade de Odontologia de Piracicaba - UNICAMP, Piracicaba, São Paulo, Brasil.

3- Professora do Departamento de Clínica e Odontologia Social, Curso de Odontologia, Universidade Federal da Paraíba - UFPB, João Pessoa, Paraíba, Brasil.

4- Professora do Programa de Pós-Graduação em Odontologia, Universidade Federal da Paraíba - UFPB, João Pessoa, Paraíba, Brasil. 
E m face ao aumento da expectativa de vida da população mundial, atualmente 71,4 anos para nascidos em 2015¹, observa-se também incremento da faixa populacional de indivíduos idosos; estima-se que, em 2020, a população mundial com mais de 60 anos superará o número de crianças menores de 5 anos $^{2}$.

Assim, se faz necessário determinar o perfil de saúde deste extrato da população, sobretudo as condições de saúde bucal, visto que grande parte destes indivíduos já passaram pela perda de algum elemento dentário, como confirmam os dados do SB Brasil 2010, com média de 29,4 dentes perdidos dentre o grupo etário de 65 a 74 anos $^{3}$. Isto reflete a necessidade de tratamento reabilitador que englobe a instalação de próteses fixas ou removíveis, visando o reestabelecimento da função mastigatória, estética e fonética ${ }^{4}$.

Fatores como higienização deficiente, próteses com muitos anos de uso, em que a degradação do material é previsível, além da pressão negativa existente na interface resina acrílica-mucosa, levam ao acúmulo de biofilme na superfície das mesmas. A ação deste biofilme, composto de variadas espécies e, dentre elas, a Candida albicans, favorece quadros infecciosos na mucosa oral. Este fungo tem sido mencionado como o mais prevalente quando do diagnóstico de estomatite associada à dentadura ${ }^{5}$.

Alguns estudos relatam o isolamento de C. albicans, tanto na mucosa oral quanto na base das próteses, caracterizando-a como nicho de colonização do biofilme. A ocorrência de C. albicans foi verificada em $36,67 \%$ das amostras de mucosa e 53,33\% nas amostras de próteses ao serem avaliados 30 portadores de estomatite protética ${ }^{6}$.

Nesse sentido, a qualidade de vida, a partir das condições de saúde bucal, e a higiene de próteses dentárias, tornam-se aspectos relevantes a serem avaliados. A análise da presença de biofilme maduro nas superfícies protéticas auxilia na construção de um planejamento e execução de programas educacionais, voltados para a prevenção e promoção dos cuidados à saúde, principalmente nesta faixa etária?

Adicionalmente, o conceito de saúde engloba o bem-estar mental, físico e social, sendo inserido neste contexto o conceito de qualidade de vida, a qual pode ser afetada por limitações sociais e funcionais ${ }^{8}$. Assim, a avaliação da qualidade de vida dos pacientes que sofreram perdas dentárias e apresentam tratamento reabilitador, considerando as condições de saúde bucal, devem ser investigadas, pois podem expressar impactos negativos à saúde.

Dessa maneira, avaliou-se a qualidade de vida e as condições de higiene de próteses dentárias em idosos institucionalizados. Incluiu-se também um levantamento sobre as instruções em saúde bucal recebidas na instituição, uma vez que o acompanhamento do cirurgião-dentista constitui um dos pilares para o alcance da higienização correta e contínua da prótese dentária.

\section{METODOLOGIA}

O estudo foi realizado no Lar da Providência Carneiro da Cunha, na Cidade de João Pessoa/ PB, no período de novembro de 2016 a abril de 2017. Foi precedido de aprovação pelo Comitê de Ética em Pesquisa do Centro de Ciências da Saúde da Universidade Federal da Paraíba, sob o CAAE 59858516.0.0000.518 e do consentimento livre e esclarecido dos participantes.

A amostra foi composta por 38 idosos institucionalizados e todos faziam uso de prótese total ou parcial removível. A princípio, para caracterização dos participantes da pesquisa, aplicou-se um formulário composto por oito questões acerca de sexo, idade, tipo de prótese, instrução de higiene oral e dos métodos utilizados para este fim.

Em seguida foi utilizado o instrumento Geriatric Oral Health Assessment Index (GOHAI), desenvolvido para avaliar a autopercepção das condições de saúde bucal e seu impacto na qualidade de vida de idosos ${ }^{9}$. As questões foram organizadas em três dimensões: física e funcional, psicossocial e estética.

Para cada pergunta foram possíveis três respostas - sempre, algumas vezes e nunca - às quais foram atribuídos, respectivamente, escores de 1 a 3 . O somatório dos escores variou de 12 a 36, por indivíduo e, então, a qualidade de vida foi classificada como: baixa, quando os escores eram menores que 30; moderada, quando de 30 a 33 e alta, quando de 34 a 36. Quanto maior o escore, melhor é a autopercepção da condição bucal e mais alta qualidade de vida ${ }^{9}$.

Dos 38 idosos que responderam ao formulário, 76,3\% $(n=29)$ concordaram em remover as próteses para avaliação da retenção de biofilme na região de palato e rebordo. Inicialmente, as próteses foram recobertas por solução evidenciadora, Eviplac Solução Biodinâmica ${ }^{\mathrm{TM}}$. Após a remoção 
do excesso, as próteses foram fotografadas, sobre fundo escuro e luz natural e, em seguida, realizada a higienização com escova dental e dentifrício. Sobre cada imagem obtida das próteses foi inserida uma malha quadriculada $1 \times 1 \mathrm{~cm}$, em imagem computacional, que, na avaliação da presença de biofilme (Figura 1), foi classificada em baixa, moderada e alta. Foram avaliadas 39 próteses no total, sendo $66,6 \%(n=26)$ superiores e $33,4 \% \quad(n=13)$ inferiores. Instruções de higiene foram fornecidas aos participantes da pesquisa.

Os dados do formulário e do GOHAI foram analisados descritiva e inferencialmente com o auxílio do software IBM SPSS na sua versão 20.0 e submetidos aos testes Qui-quadrado e Exato de Fisher $(\alpha=0,05)$. A qualidade de vida foi agrupada em alta e moderada-baixa, sendo considerada a variável de desfecho.

\section{RESULTADOS}

Os participantes do estudo tinham idade média de 86,8 \pm 7,7 anos, dentre eles, $86,8 \%$ $(n=33)$ eram do sexo feminino e $92,1 \% \quad(n=35)$ faziam uso de prótese total.

Dos indivíduos que compuseram a amostra, $86,8 \%(n=33)$ afirmaram não remover a prótese em nenhum período do dia e, destes, $63,2 \%(n=24)$ também não retiravam para dormir. Quando questionados sobre o tempo de uso da prótese atual, $63,2 \%(n=24)$ afirmaram fazer uso da mesma há mais de 5 anos. Os idosos foram também questionados quanto ao fato de já terem recebido, na instituição, instrução sobre a higienização correta de suas próteses; $65,7 \% \quad(n=25)$ afirmaram que não. O método de higienização mecânico foi citado por $97,4 \%(n=37)$. Já 94,7\% $(n=36)$ não realizam nenhum tipo de higienização química.

A qualidade de vida foi classificada, em sua maioria, como alta (Figura 3; Tabela 1). O somatório dos escores atribuídos para cada resposta do GOHAl variaram de 22 a 36 , sendo 34 o mais frequente, representado por $44,7 \%(n=17)$ da amostra.

Com relação à satisfação quanto à aparência da boca, $65,8 \%(n=25)$ referiram estar sempre satisfeitos. Quando questionados sobre sentir dor ou desconforto ao comer ou engolir $73,7 \%$ $(n=28)$ da amostra relatou que não. Quanto da associação entre os questionamentos sobre o uso e higienização das próteses e a qualidade de vida, observou-se associação $(p=0,001)$ entre o fato dos idosos não retirarem a prótese para dormir e ter uma alta qualidade de vida, segundo o GOHAI. Fatores como fazer uso da mesma prótese há mais de 5 anos, receberem acompanhamento odontológico, instruções sobre a correta higienização das próteses, entre outros, não tiveram impacto na qualidade de vida dos participantes desta pesquisa.

Em relação à análise da presença de biofilme nas superfícies protéticas, dentre as 26 próteses superiores coradas e analisadas, a maioria apresentou retenção de baixa a moderada, tanto para área de rebordo $(n=23)$ quanto para área de palato $(n=25)$. Entre as 13 próteses inferiores analisadas, em $84,6 \%(n=11)$ delas a retenção se mostrou moderada a alta.

Figura 1: Próteses coradas com solução evidenciadora e colocadas sobre fundo escuro para posterior fotografia e análise sob malha quadriculada $1 \times 1 \mathrm{~cm}$.
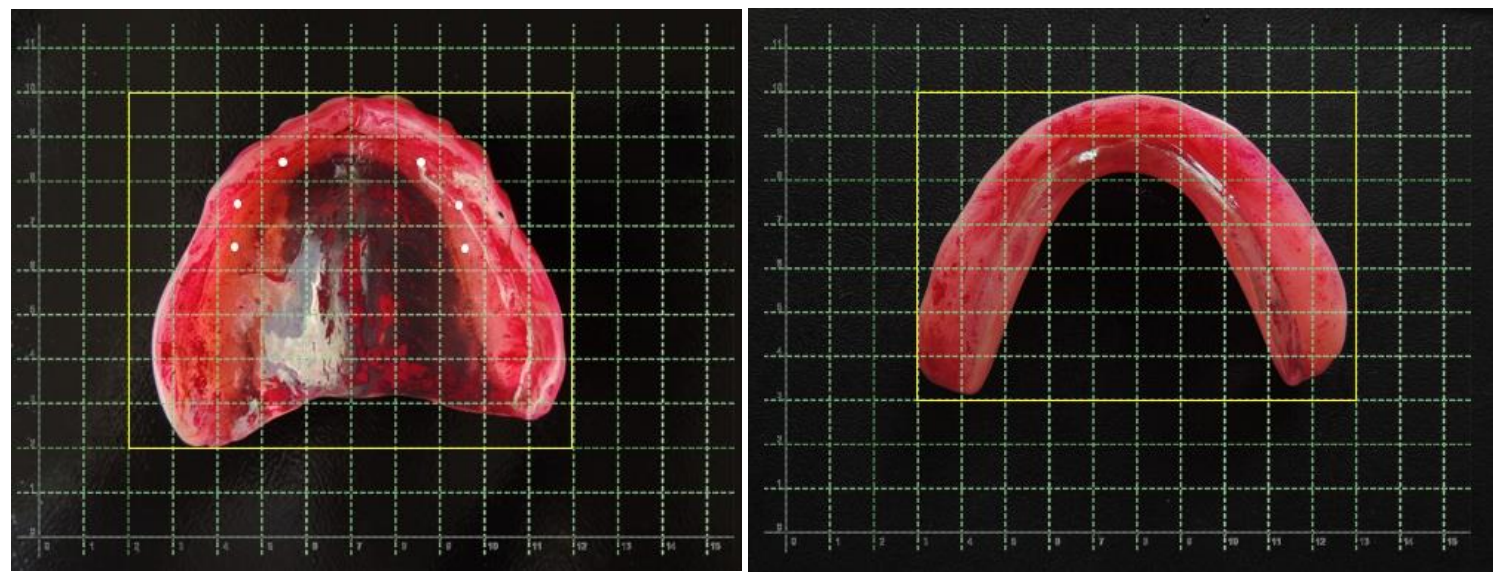
Figura 2: Distribuição por faixa etária dos idosos usuários de prótese dentária do Lar da Providência Carneiro da Cunha, João Pessoa/PB, 2016.

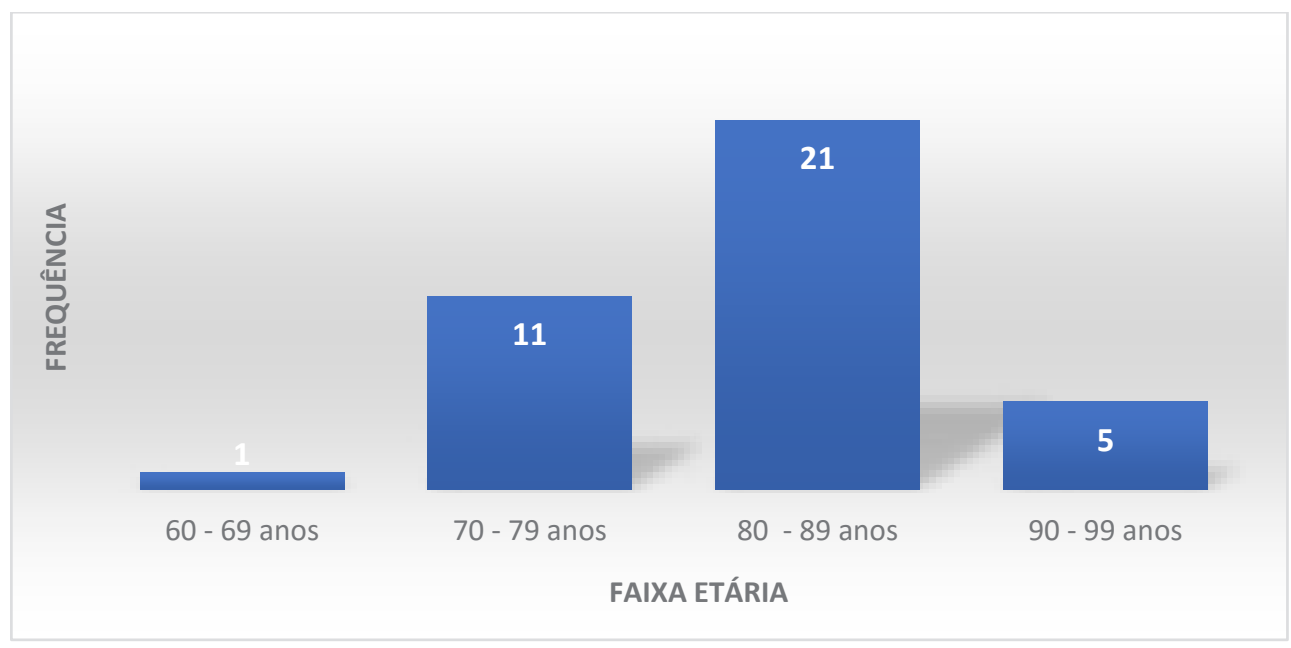

Figura 3: Distribuição da frequência por classificação quanto à qualidade de vida de idosos usuários de prótese dentária do Lar da Providência Carneiro da Cunha, João Pessoa/PB, 2016.

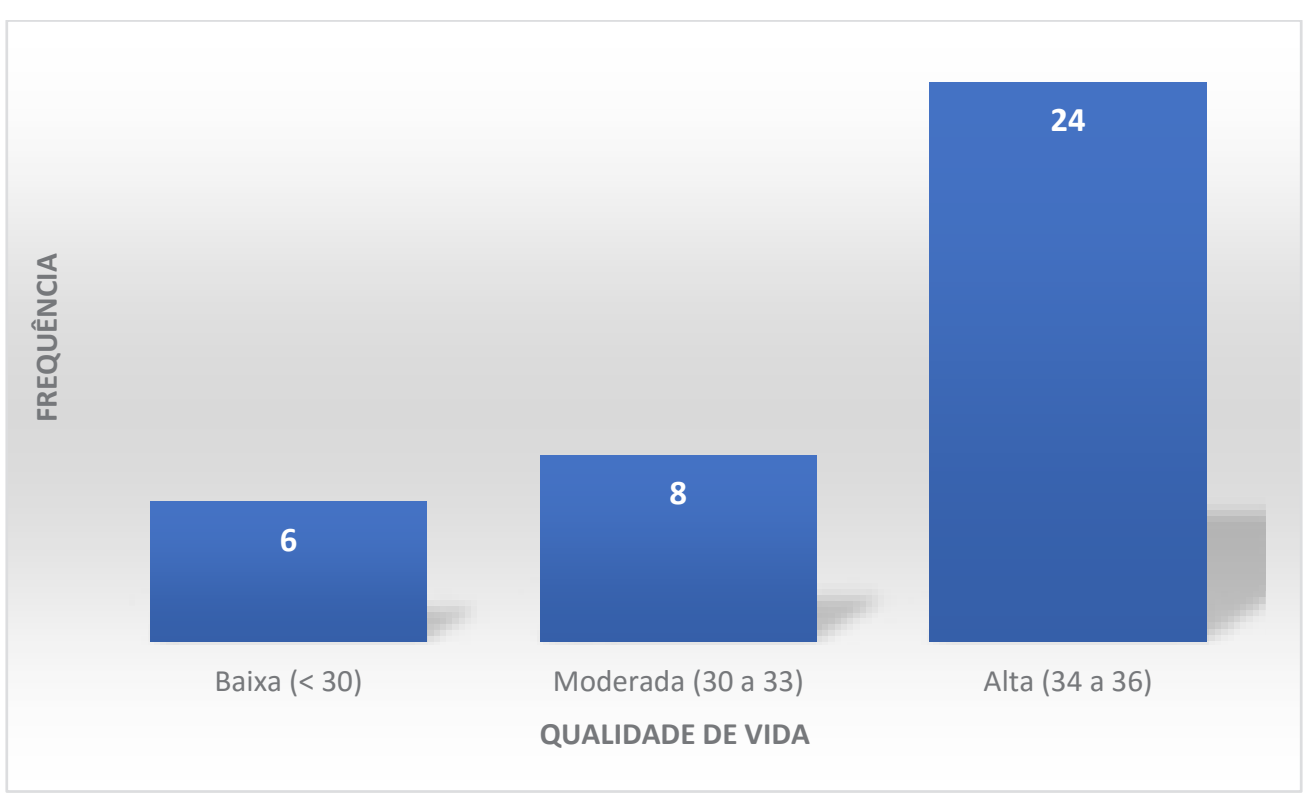


Tabela 1: Associação entre a autopercepção da saúde bucal a partir do índice GOHAl e algumas características dos idosos usuários de prótese dentária do Lar da Providência Carneiro da Cunha, João Pessoa/PB, 2016.

\begin{tabular}{|c|c|c|c|c|c|c|c|}
\hline & \multicolumn{4}{|c|}{ Qualidade de Vida } & \multirow{3}{*}{$\begin{array}{l}\text { Total } \\
\%(n)\end{array}$} & \multirow{3}{*}{$p$} \\
\hline & & \multicolumn{2}{|c|}{ Alta } & \multicolumn{2}{|c|}{ Moderada/Baixa } & & \\
\hline & & $n$ & $\%$ & $\mathrm{n}$ & $\%$ & & \\
\hline \multirow[t]{2}{*}{ Tipo de Prótese } & Total & 21 & 87,5 & 14 & 100 & $\begin{array}{l}92,1 \\
(35)\end{array}$ & \multirow[t]{2}{*}{,283* } \\
\hline & Parcial & 3 & 12,5 & 0 & 0 & $7,9(3)$ & \\
\hline \multirow{2}{*}{$\begin{array}{l}\text { Higienização } \\
\text { Mecânica }\end{array}$} & Sim & 23 & 95,8 & 14 & 100 & $\begin{array}{l}97,4 \\
(37)\end{array}$ & \multirow[t]{2}{*}{$1,000^{*}$} \\
\hline & Não & 1 & 4,2 & 0 & 0 & $2,6(1)$ & \\
\hline \multirow{2}{*}{ Higienização Química } & Sim & 0 & 0 & 2 & 14,3 & $5,3(2)$ & \multirow{2}{*}{$0,129^{*}$} \\
\hline & Não & 24 & 100 & 12 & 85,7 & $94,7(36)$ & \\
\hline \multirow{2}{*}{$\begin{array}{l}\text { Remove Prótese em } \\
\text { Período do Dia }\end{array}$} & Sim & 2 & 8,3 & 3 & 21,4 & $13,2(5)$ & \multirow{2}{*}{$0,337^{*}$} \\
\hline & Não & 22 & 91,7 & 11 & 78,6 & $86,8(33)$ & \\
\hline \multirow{2}{*}{$\begin{array}{l}\text { Remove a Prótese } \\
\text { para Dormir }\end{array}$} & Sim & 4 & 16,7 & 10 & 71,4 & $36,8(14)$ & \multirow{2}{*}{$0,001^{*}$} \\
\hline & Não & 20 & 83,3 & 4 & 28,6 & $63,2(24)$ & \\
\hline \multirow{2}{*}{$\begin{array}{l}\text { Mesma Prótese há } \\
\text { mais de } 5 \text { anos }\end{array}$} & Sim & 17 & 70,8 & 7 & 50 & $63,2(24)$ & \multirow{2}{*}{0,199} \\
\hline & Não & 7 & 29,2 & 7 & 50 & $36,8(14)$ & \\
\hline \multirow{2}{*}{$\begin{array}{l}\text { Instrução de Correta } \\
\text { Higiene da Prótese }\end{array}$} & Sim & 6 & $25 \%$ & 7 & $50 \%$ & $34,2(13)$ & \multirow{2}{*}{0,117} \\
\hline & Não & 18 & $75 \%$ & 7 & $50 \%$ & $65,8(25)$ & \\
\hline \multirow{2}{*}{$\begin{array}{l}\text { Acompanhamento } \\
\text { Odontológico na } \\
\text { Instituição }\end{array}$} & Sim & 4 & 16,7 & 3 & 21,4 & $18,4(7)$ & \multirow{2}{*}{$1,000^{*}$} \\
\hline & Não & 20 & 83,3 & 11 & 78,6 & $81,6(31)$ & \\
\hline
\end{tabular}




\section{DISCUSSÃO}

Uma das primeiras observações, a partir do edentulismo, é a alteração estética, todavia, implicações na fonação e na função mastigatória vem também acompanhadas da perda dentária. Certos fonemas, como o labiodental, linguodental ou linguo-alveolar, requerem a contribuição dos dentes para sua construção ${ }^{10}$. Dada à perda, o indivíduo pode apresentar dificuldade e, às vezes, impossibilidade de reproduzir corretamente as palavras.

A falta dos dentes afeta também a capacidade mastigatória, o que pode levar a uma mudança nos hábitos alimentares, quando o indivíduo precisa optar por alimentos mais macios e menos fibrosos. Tais alimentos podem possuir menor valor nutritivo, o que demonstra a importância da reabilitação desses pacientes ${ }^{11}$.

Os participantes da presente pesquisa são reabilitados, ou seja, receberam tratamento que Ihes devolveu função. Com isso, o objetivo do estudo diz respeito à orientação acerca dos cuidados orais, uma vez que a má higienização, seja pela falta de instrução ou de destreza manual, devido ao avanço da idade e à presença de distúrbios motores, afeta a condição de saúde bucal, o que favorece o aparecimento de processos inflamatórios, como a estomatite protética e infecções fúngicas, como a candidose oral ${ }^{12}$. Foi possível verificar que idosos que relatavam dificuldade de higiene e não realizavam nenhum tipo de higienização química, apresentavam próteses com maior quantidade de biofilme aderido, portanto, mais susceptíveis ao surgimento de patologias orais e maior risco de infecções.

A estomatite protética é uma condição que acomete a mucosa do palato devido ao contato direto com a prótese removível, total ou parcial. Quando não tem etiologia traumática, seus fatores desencadeantes são aqueles que favorecem o estabelecimento de biofilme, dentre eles: idade avançada associada ao declínio da atividade do sistema imunológico, doenças sistêmicas, tabagismo, uso da prótese para dormir e higiene insatisfatória ${ }^{13-15}$.

Embora não tenha sido objetivo do pre- sente estudo diagnosticar a presença de estomatite protética, observou-se que a maioria dos participantes não removem a prótese em nenhum período do dia ou da noite, tornando-se indivíduos com potencial risco de desenvolvimento desta condição. Além disto, apenas 34,2\% $(n=13)$ afirmaram já terem sido instruídos quanto à higienização, o que Ihes coloca, então, numa situação sensível ao estabelecimento da patologia ${ }^{13}$

Corroborando com um outro estudo ${ }^{16}$, que apontou bom score GOHAl para $44,7 \%$ dos 857 idosos que compunham sua amostra, os dados obtidos nesta pesquisa indicam que a percepção e a qualidade de vida dos indivíduos são elevadas. Os mesmos mostram-se satisfeitos com a aparência da boca e não apresentam dor ou desconforto ao comer ou engolir alimentos consistentes, demonstrando uma perspectiva real da situação dos idosos que já estão reabilitados. Achados semelhantes foram obtidos em um outro estudo ${ }^{17}$ no qual 166 idosos residentes em instituições de longa permanência do Rio Grande do Norte foram questionados sobre a autopercepção de saúde bucal e $65 \%(n=108)$ deles indicaram como boas ou excelentes as condições dos dentes, gengiva e próteses.

Observou-se que a percepção da qualidade de vida foi considerada alta naqueles que não removiam a prótese ao deitar. Este fato pode estar associado principalmente à dimensão estética abordada pelo GOHAl. É provável que, para considerar percepção alta, os indivíduos tenham atribuído mais valor à aparência da boca do que à possível dor ou desconforto causados pelo uso da peça. Portanto, idosos que dormem com a prótese estão mais satisfeitos com sua condição bucal, diferente daqueles que dormem sem ela.

Estes achados divergem dos resultados obtidos em um estudo realizado num Centro de Recreação da Cidade do México ${ }^{18}$, com 150 idosos atendidos regularmente. Observou-se que $84 \%$ $(n=126)$ deles relataram estar descontentes com a aparência da boca, 90,6\% $(n=136)$ referiram desconforto ao comer, $66 \%(n=99)$ mencionaram problemas ao mastigar e $64 \%(n=96)$ disseram ter alterado o tipo de alimento ingerido. 
Quanto à mensuração de biofilme nas próteses, as áreas de rebordo ou palato apresentaram quantidade de acúmulo semelhante - quando baixa, moderada ou alta em rebordo, o resultado se repetia para área de palato. As próteses superiores, de modo geral, foram classificadas como tendo retenção de baixa a moderada. As próteses inferiores se mostraram mais retentivas, por apresentarem adesão moderada a alta, em sua maioria. Isso se dá, possivelmente, pela dificuldade de acesso, com a escova, nas depressões que existem para acomodar o rebordo inferior, que por sua vez, poderia ser compensada com a higienização química.

Resultados positivos quanto à eficácia do hipoclorito de sódio na viabilidade celular de espécies de Candida nas superfícies de próteses dentárias são observados na literatura ${ }^{19}$. Estudos in vitro mostram que o uso do hipoclorito de sódio a $0,5 \%$ auxilia na desinfecção das próteses, e a incorporação de nistatina nesses materiais, também é capaz de tratar ou prevenir a candidíase oral ${ }^{20}$. Entretanto, o presente estudo verificou que o método químico de higienização não é realizado por $94,7 \%(n=36)$ dos participantes.

A principal limitação deste estudo é relativa ao tamanho da amostra pesquisada. Por se tratar

\section{REFERÊNCIAS}

1. WORLD HEALTH ORGANIZATION. World health statistics 2017: monitoring health for the SDGs, Sustainable Development Goals. Geneva, 2017.

2. WORLD HEALTH ORGANIZATION. "Ageing well" must be a global priority. 06 de novembro, 2014. Disponível em: http://www.who.int/mediacentre/news/releases/2014/lancet-ageing-series/en/. Acesso em: 01 de agosto de 2017.

3. BRASIL. Ministério da Saúde. SB Brasil 2010: Pesquisa Nacional de Saúde Bucal: resultados principais. Brasília: Ministério da Saúde, 2012.

4. Gennari-Filho, HO. Exame clínico em prótese total. Rev. Odontol. Araçatuba. 2004; 25(2): 62-71.

5. Gauch LMR, Pedrosa SS, Silveira-Gomes F, Esteves $\mathrm{RA}$, Marques-da-Silva SH. Isolation of Candida spp. from denture-related stomatitis in Pará, Brazil. Braz. J. Microbiol. 2018; 49(1): 149-51.

6. Leite DP, Piva MR, Martins-Filho PRS. Identificação das espécies de Candida em portadores de estomatite protética e avaliação da susceptibilidade ao miconazol e à terapia fotodinâmica. Rev. Odontol. UNESP. 2015; 44(1): 12-17. de um estudo exploratório, os resultados aqui descritos dizem respeito somente aos idosos residentes na instituição em questão e não podem ser representativos para toda João Pessoa ou Estado, principalmente para os idosos que apresentam autonomia e residem em domicílios. Entretanto, por considerar cada vez maior o aumento desta faixa etária da população, sugere-se novos estudos que englobem uma amostra ampliada, bem como outros aspectos relacionados não somente à saúde bucal, mas, como por exemplo, o estado de saúde geral destes indivíduos.

\section{CONCLUSÃO}

A alta qualidade de vida esteve associada ao fato do idoso não retirar a prótese para dormir. Entretanto, fatores como período de utilização da prótese ou conhecimentos sobre métodos de higienização não influenciaram a qualidade de vida.

Com relação à retenção de biofilme, as próteses inferiores apresentaram maior área de retenção que as superiores. Além disto, os indivíduos relataram não receber instrução sobre higiene das próteses e saúde bucal ou acompanhamento por parte de cirurgião-dentista na instituição.

7. Silva SRC, Rosell FL, Valseki-Junior A. Percepção das condições de saúde bucal por gestantes atendidas em uma unidade de saúde no município de Araraquara, São Paulo, Brasil. Rev. Bras. Saúde Mater. Infant. 2006; 6(4): 405-10.

8. Zenthöfer A, Rammelsberg P, Cabrera T, Schröder J, Hassel AJ. Determinants of oral health-related quality of life of the institutionalized elderly. Psychogeriatrics. 2014; 14(4): 247-54.

9. Carvalho C, Manso AC, Escoval A, Salvado F, Nunes C. Tradução e validação da versão portuguesa do Geriatric Oral Health Assessment Index (GOHAI). Rev. Port. Saúde Pública. 2013; 31(2): 153-59.

10. Özbeki M, Tulunoglu Í, Özkan S, Öktemer M. Evaluation of articulation of Turkish phonemes after removable partial denture application. Braz. Dent. J. 2003; 14(2): 125-31.

11. Campos MTFS, Monteiro JBR, Ornelas APRC. Fatores que afetam o consumo alimentar e a nutrição do idoso. Rev. Nutr. 2000; 13(3): 157-65. 
12. Gonçalves LFF, Neto DRS, Bonan PRF, Carlo HL, Batista AUD. Higienização de Próteses Totais e Parciais Removíveis. Rev. Bras. Ciênc. Saúde. 2011; 15(1): 87-94.

13. Evren BA, Uludamar A, Iseri U, Ozkan YK. The association between socioeconomic status, oral hygiene practice, denture stomatitis and oral status in elderly people living different residential homes. Arch. Gerontol. Geriatr. 2011; 53(3): 252-57.

14. Gendreau L, Loewy ZG. Epidemiology and etiology of denture stomatitis. J. Prosthodont. 2011; 20(4): 252-60.

15. Vasconcelos LC, Vasconcelos LCS, Ghersel ELA, Veloso DJ, Cunha PASNA. Denture hygiene: importance in denture stomatitis control. Rev. Gaúch. Odontol. 2013; 61(2): 255-61.

16. De Andrade FB, Lebrão ML, Santos JLF, Teixeira DSC, Duarte YAO. Relationship Between Oral Health-Related Quality of Life, Oral Health, Socioeconomic, and General Health Factors in Elderly Brazilians. J. Am. Geriatr. Soc. 2012; 60(9): 1755-60.

17. Melo LA, Sousa MM, Medeiros AKB, Carreiro AFP, Lima $\mathrm{KC}$. Fatores associados à autopercepção negativa da saúde bucal em idosos institucionalizados. Ciênc. Saúde Colet. 2016; 21(11): 339-346.
18. Hernández-Palacios RD, Ramírez-Amador V, Jarillo-Soto EC, Igoyen-Camacho ME, Mendonza-Núñez VM. Relationship between gender, income and education and self-perceived oral health among elderly Mexicans. An exploratory study. Ciênc. Saúde Colet. 2015; 20(4): 997-1004.

19. Vieira AP, Senna PM, Silva WJ, Del Bel Cury AA. Longterm efficacy of denture cleansers in preventing Candida spp. biofilm recolonization on liner surface. Braz. Oral Res. 2010; 24(3): 342-48.

20. Skupien JA, Valentine F, Boscato N, Pereira-Cenci T. Prevention and treatment of Candida colonization on denture liners: A sistematic review. J. Prosthet. Dent. 2013; 110(5): 356-62.

\section{CORRESPONDÊNCIA}

Ana Maria Gondim Valença

Av, Miguel Sátyro, 350/2301 - Cabo Branco, João Pesssoa/PB CEP 58.045-110

Email: anamvalenca@gmail.com 